\title{
CORRECTIONS
}

\section{Unintended consequences of sanctions against Iran}

In this Feature (BMJ 2013;347:f4650, doi:10.1136/bmj.f4650) we incorrectly stated that Behrooz Astaneh's position at the Iranian Journal of Medical Sciences is "acting editor," when Cite this as: BMJ 2013;347:44787 he is in fact editor. 